\title{
Ecrire l'histoire du Commonwealth des Nations:relations internationales et dialogues disciplinaires
}

Writing the History of the Commonwealth of Nations: International Relations and Disciplinary Dialogue

Mélanie Torrent

\section{OpenEdition}

Journals

Édition électronique

URL : http://journals.openedition.org/rfcb/2946

DOI : $10.4000 /$ rfcb.2946

ISSN : 2429-4373

Éditeur

CRECIB - Centre de recherche et d'études en civilisation britannique

Référence électronique

Mélanie Torrent, «Ecrire l'histoire du Commonwealth des Nations:relations internationales et dialogues disciplinaires », Revue Française de Civilisation Britannique [En ligne], XXIV-1 | 2019, mis en ligne le 22 mars 2019, consulté le 01 mai 2019. URL : http://journals.openedition.org/rfcb/2946 ; DOI : $10.4000 /$ rfcb.2946

Ce document a été généré automatiquement le 1 mai 2019.

\section{cc) (†) $\odot$}

Revue française de civilisation britannique est mis à disposition selon les termes de la licence Creative Commons Attribution - Pas d'Utilisation Commerciale - Pas de Modification 4.0 International. 


\title{
Ecrire l'histoire du Commonwealth des Nations:relations internationales et dialogues disciplinaires
}

\author{
Writing the History of the Commonwealth of Nations: International Relations \\ and Disciplinary Dialogue
}

Mélanie Torrent

\section{Introduction}

1 Le Commonwealth des Nations, affirmait le chef Emeka Anyaoku, l'un de ses anciens Secrétaires généraux, lors d'un discours au club de la Royal Overseas League à Londres en juillet 2010, demeure "une organisation atypique et asymétrique», et un "exemple global de compréhension et de coopération » qui, s'il demande certes à être amélioré, porte néanmoins le respect de la diversité au cœur même de ses principes. Face à un public composé d'universitaires, de journalistes, de diplomates et de membres d'organisations non-gouvernementales, mais aussi de plusieurs anciens administrateurs de l'empire britannique, Anyaoku estimait qu'il était « important d'envisager l'avenir du Commonwealth, non pas à travers le prisme d'un passé impérial mais comme une organisation nouvelle, en expansion, capable de déployer ses qualités uniques pour accomplir un service global ${ }^{1} »$. Si les propos d'Anyaoku reflètent les paradoxes du Commonwealth des Nations contemporain, ils incitent également à une réflexion sur l'articulation entre empires et fins d'empire, diplomaties officielles et imaginaires sociétaux. Les multilatéralismes en œuvre au sein du Commonwealth, organisation internationale qui n'est pas qu'une simple réitération ou un long prolongement de l'empire britannique, posent les connections (de structures, de pensée, de personnes) comme objet et outil d'étude, notamment entre l'ensemble du réseau Commonwealth et 
les multiples nations et Etats qui le composent, et entre le Commonwealth lui-même et les « autres » Commonwealth, au premier rang desquels la Francophonie.

D’emblée, l'histoire du Commonwealth institutionnel est nécessairement fondée sur un double dialogue, entre disciplines universitaires et entre aires culturelles, répondant à un double défi. D'une part, comme le suggérait récemment Bertrand Badie dans son ouvrage sur les évolutions de la diplomatie, les "grandes questions sociales internationales se révèlent beaucoup plus structurantes et déterminantes que les enjeux géostratégiques » et font que les Etats sont peut-être « de plus en plus promis à réagir à la dynamique des sociétés, plutôt qu'à agir sur elles ${ }^{2}$ ». Une telle perspective souligne l'importance de l'histoire connectée dont le principal apport est cette "volonté de décloisonner en articulant le social, l'économique, le culturel et le politique [pour] restituer à la fois l'épaisseur du jeu social et la globalité des échanges qui l'animent ${ }^{3}$ ». Mais elle confirme aussi la nécessité d'un dialogue interdisciplinaire plus soutenu, les questions de politique étrangère, diplomatie et relations internationales ne pouvant se comprendre indépendamment des environnements politiques, économiques, culturels, sociaux et sociétaux des pays considérés. D'autre part, l'étude de l' « objet » Commonwealth ne peut faire l'économie d'une connaissance des pays qui en sont membres - mais le nombre même de ces territoires est un défi en soi. Le risque majeur est de s'intéresser avant tout aux « grands » du Commonwealth (Royaume-Uni, Australie, Canada, Afrique du Sud, Inde et Nigéria, notamment) mais l'autre risque est de généraliser à partir de grandes régions ou continents (l'Afrique par exemple, en ignorant la grande diversité entre le Malawi et le Kenya, par exemple, ou entre le Rwanda et la Sierra Leone; ou les Caraïbes, où la Jamaïque n'est pas Sainte-Lucie). Le dialogue entre les spécialistes des diverses aires qui le composent et entre disciplines universitaires est donc au cœur même de l'histoire du Commonwealth - le Commonwealth comme «terrain » pour le chercheur étant à la fois vaste, adaptable et nécessairement au final circonscrit par les points d'entrée disciplinaires et géographiques choisis.

$\mathrm{Au}$ début des années 1990 Shula Marks, l'historienne sud-africaine qui dirigeait alors l'Institute of Commonwealth Studies à l'Université de Londres, regrettait que les historiens du Commonwealth soient essentiellement des hommes, blancs, marginalisant davantage l'histoire dans les disciplines prisées au sein des "Commonwealth studies " ${ }^{4}$. Si l'histoire de l'empire a su faire, toutes proportions gardées, une place plus grande à la question des femmes et du genre, l'histoire des décolonisations fait face à de nombreux chantiers, encore très peu travaillés 5 . Le Commonwealth est un paramètre - et un acteur central de l'histoire contemporaine du Royaume-Uni, notamment pour l'analyse de l'influence britannique dans le monde et de l'impact des fins d'empire sur l'Etat et la société britanniques : l'unique langue officielle de l'organisation est l'anglais ; si depuis la Déclaration de Londres de 1949, le monarque britannique est uniquement le chef symbolique du Commonwealth, le choix du Prince Charles comme successeur d'Elizabeth II lorsque l'heure sera venue en conforte l'ancrage britannique; le Secrétariat du Commonwealth, parfaitement indépendant du Royaume-Uni depuis sa création en 1965, demeure localisé à Malborough House à Londres; et l'entrée de membres non-issus de l'ancien empire britannique, notamment le Rwanda en $2009^{6}$, reste, ainsi que l'indiquent les textes officiels, un fait exceptionnel.

Cet article propose non pas de retracer l'histoire du Commonwealth contemporain mais plutôt de montrer comment le dialogue entre disciplines a permis un renouvellement de l'écriture de cette histoire, et dans quelle mesure le Commonwealth des Nations, comme 
espace géopolitique, acteur international multilatéral et objet d'imaginaires politiques divers, constitue un prisme d'analyse critique pertinent pour l'histoire - internationale, transnationale et connectée - du monde contemporain.

\section{Le Commonwealth, entre théorie et histoire des relations internationales}

Dans l'historiographie des fins d'empire, le Commonwealth mérite d'occuper une place plus importante, à la fois comme aire géographique - aux réalités très variées et au périmètre variable, selon les périodes considérées - et comme concept - objet de vives disputes sous toutes ses formes, des débats sur une fédération impériale au tournant du $\mathrm{XX}^{\mathrm{e}}$ siècle aux controverses sur l'admission de membres non-issus de l'empire britannique au tournant du siècle actuel et, tout récemment, à sa pertinence pour un Royaume-Uni à la recherche d'une nouvelle identité globale à l'heure du «Brexit». Il ne s'agit pas d'en faire une sphère omniprésente dans les préoccupations des décideurs ou d'accorder aux relations multilatérales une importance excessive, alors que les bilatéralismes, au sein même du Commonwealth, ont souvent occupé une place prépondérante ${ }^{7}$. Mais comme le suggèrent les travaux de Mark Mazower, l'idée d'un "commonwealth des nations" se retrouve au-delà des réseaux constitués du Commonwealth britannique et de ses incarnations ultérieures, qui partagent avec d'autres projets politiques l'ambition de lier nationalisme et internationalisme. De fait, les débats sur le concept même de nation, au sein d'un Commonwealth des "nations" désormais composé en réalité d'Etats, confirment les limites de cette notion pour comprendre « les communautés imaginées » au sein de systèmes culturels qui soulignent les inadéquations entre territoire et nation dans de très nombreux cas. Pour le Commonwealth des Nations comme pour les autres projets politiques, les absences, échecs et projets alternatifs sont tout aussi importants que les mises en œuvre, parce qu'ils permettent justement de mieux expliquer pourquoi et comment ce sont ces mises en œuvre-là qui ont été choisies, et de situer « conceptuellement et historiquement dans un contexte international » le nationalisme, à la fois « comme idéologie et comme forme de pratique politique ${ }^{8} »$.

6 Qu'il soit compris comme construction impériale ou comme acteur international, et parce qu'il ne fonctionne jamais en espace clos, le Commonwealth permet aussi de ne pas faire de la décolonisation une simple catégorie de l'histoire britannique mais une manière d'appréhender l'histoire britannique depuis l'histoire globale et connectée. Pour Ian Hall, la décolonisation, bien loin de rencontrer l'indifférence des penseurs britanniques, a eu une influence notable sur la pensée internationale au Royaume-Uni, depuis les divers courants de l'école anglaise des relations internationales jusqu'à la Nouvelle Gauche. Pour ceux qui voyaient dans l'empire un moyen de progrès (tout critiquable que fût l'impérialisme), la décolonisation semblait mettre à mal leurs idéaux internationalistes libéraux, tout comme pour ceux qui pensaient avant tout l'empire comme un moyen de structuration du monde et d'ordre international. Hedley Bull s'inquiétait ainsi non pas du fait de la décolonisation, mais de ses résultats sur l'équilibre du monde et les régimes normatifs internationaux. En parallèle, plusieurs penseurs britanniques soulignaient qu'il n'y avait en réalité que peu de changement et que la « révolte contre l'occident » décrite par Bull n'avait pas empêché les «normes, intérêts et objectifs des pouvoirs hégémoniques - la promotion de la paix, la prospérité et les "critères de civilisation", y 
compris la démocratie, les droits de l'homme et la protection de l'environnement - de triompher ${ }^{9} »$. Source de satisfaction pour certains - à l'image d'Adam Watson, figure importante du Foreign office et ancien ambassadeur en Afrique francophone dans les années 1960 - ces continuités firent émerger un nouveau radicalisme, cristallisé dans la Nouvelle Gauche, embrassant les anticolonialismes, les tiers-mondismes, les luttes contre les diverses formes de néocolonialismes, et soulignant les multiples violences des empires et fins d'empire à l'appui de penseurs tel Frantz Fanon.

7 Si la théorie des relations internationales a toute sa place dans le champ de l'histoire du Commonwealth, c'est précisément aussi parce que les penseurs de langue anglaise ont joué un rôle fondamental dans l'élaboration des théories des relations internationales qui a accompagné les évènements et évolutions du monde passé et présent. L'étude des organisations internationales contemporaines, dont le Commonwealth des Nations, ne peut faire l'économie d'une connaissance fine des travaux des principaux penseurs de l'école anglaise des relations internationales, de Martin Wight (1913-1972) et Hedley Bull (1932-1985) à, plus récemment, James Mayall, Barry Buzan, Nicholas Wheeler ou encore Robert Jackson. Au-delà de l'étude des mécanismes de fonctionnement des institutions internationales, l'école anglaise des relations internationales encourage également à comprendre la place du Royaume-Uni dans le monde dans la lignée de l'histoire des idées, en parallèle de l'histoire de l'étude de la coopération internationale ou même transnationale. Dans un ouvrage paru en 2009, l'historien néo-zélandais David W. McIntyre analyse l'influence d'historiens (et plus généralement peut-être, de spécialistes de divers champs des sciences humaines et de diverses «élites » politico-universitaires) sur la constitution du Commonwealth dans sa version «Britannic $»^{10}$, celle de la plus grande sphère de la Grande-Bretagne et de ses dominions de l'époque (celle de la «plus grande Grande-Bretagne ", comme certains parlent, dans d'autres contextes, de la "plus grande France »). Tout comme les écrits de l'Australien Keith Hancock, de l'Irlandais Nicholas Mansergh ou encore du Britannique Reginald Coupland permettent de mieux comprendre l'émergence du Commonwealth comme champ d'action et champ d'études entre 1907 et 1948, de même les écrits de l'école « anglaise » des relations internationales posent la question de savoir dans quelle mesure le Commonwealth peut être envisagé comme un facteur structurant de la théorisation et de la pratique des relations internationales dans la période suivante. Ceci ne revient pas à dire, ni même à postuler, qu'il y aurait un lien nécessaire entre l'étude scientifique des facteurs, modalités et conséquences de la coopération internationale et le Commonwealth comme projet politique - au risque de faire de ce dernier une lecture téléologique, déterministe et idéaliste. Mais il s'agit bien de s'interroger sur les circulations au sein des espaces, comme y encourage le dialogue théorique entre l'Australien Hedley Bull, le Canadien Robert Jackson et le Britannique James Mayall, un des spécialistes les plus reconnus de l'histoire politique du Commonwealth contemporain, au-delà de ses travaux sur le système international global.

\section{Renouvellement de l'histoire impériale - et du Commonwealth}

8 En lien avec les nouvelles histoires impériales, l'histoire du Commonwealth a fait une place plus importante à deux grandes problématiques, brisant ainsi un récit un peu trop aseptisé des fins de l'empire britannique. Le Commonwealth comme espace doit ainsi se 
penser à partir des migrations multidirectionnelles qui ont parcouru l'empire et continuent de traverser le Commonwealth contemporain, et à partir des autres espaces qui ont influencé sa structuration politique (si ce n'est institutionnelle) à l'heure des luttes pour les indépendances, y compris l'espace atlantique incluant les Etats-Unis. Les contributions rassemblées par Andrew Thompson en 2012 réfléchissent ainsi à la manière dont les géographies « impériales » et « nationales » s'imbriquaient, en travaillant sur les distinctions entre "espaces» et "lieux». Thompson soulignait bien que l'influence de l'empire n'était qu'une influence parmi bien d'autres, mais les chapitres de l'historienne Wendy Webster et du sociologue Krishnan Kumar sur les migrations, tout comme ceux de Philip Murphy sur la puissance, Richard Whiting sur la vie politique et institutionnelle et Jim Tomlinson sur l'économie confirmaient bien que la fabrique de la Grande-Bretagne au $\mathrm{XX}^{\mathrm{e}}$ siècle ne pouvait se comprendre hors d'une analyse fine et précise de l'incidence des fins d'empire dans les espaces matériels et imaginaires de l'ancienne métropole. De même, "Making Britain: how Asians shaped the nation, 1870-1950", le projet de recherche interdisciplinaire piloté en 2007-2010 par Susheila Nasta, professeur de littérature, a donné lieu non seulement à des conclusions et expositions fascinantes, mais à des bases de données extrêmement précieuses pour les chercheurs en sciences humaines et sociales ${ }^{11}$.

9 Dans la réflexion sur l'histoire intellectuelle, sociale et internationale des décolonisations, le rôle du transnationalisme comme mode de mobilisation (radicale ou conservatrice) et comme échelle d'analyse a occupé une place grandissante. Les défis méthodologiques demeurent nombreux, notamment en raison de la difficulté de combiner l'étude des personnes, marchés, flux, biens, imaginaires ou encore des idées, mais aussi parce que s'il est relativement possible d'évaluer ce qui se produit au niveau local ou global, les outils font encore défaut pour saisir les transformations qui se produisent à l'échelle même du transnational. Plusieurs propositions, avancées notamment par des chercheurs américains spécialistes des migrations comme Nina Glick Schiller, ouvrent de nouvelles perspectives stimulantes. Si les dangers du nationalisme méthodologique sont nets, les Etats, comme entité politique et territoriale, demeurent des objets d'analyse - et d'action - importants. Là où les études sur les mobilités («mobility studies») ont eu tendance à privilégier un cosmopolitisme libérateur et les parcours des élites, marginalisant davantage les populations déjà marginalisées par ces processus migratoires, Nina G. Schiller et Noel B. Salazar utilisent le concept de «régimes de mobilité », en plus de ceux de gouvernementalité et d'hégémonie, pour analyser l'impact des Etats et administrations sur les mobilités individuelles. Les questions qu'ils posent sur la normalisation de la mobilité et de l'immobilisme, et sur «la manière dont certains développements dans l'économie politique globale d'une ère spécifique forment et/ou sont reflétés dans la théorie sociale dominante ${ }^{12} »$ sont tout aussi pertinentes pour l'étude des transnationalismes de fins d'empire et pour les nouvelles conceptions des politiques étrangères, notamment (mais pas exclusivement) en lien avec les diasporas.

Le transnationalisme, y compris dans les interstices que les méthodologies actuelles n'expliquent pas totalement, ouvre ainsi de nouvelles perspectives sur les relations au sein du Commonwealth, ainsi qu'avec d'autres réseaux de solidarités (politiques, comme les entreprises tiers-mondistes, ou religieuses, entre autres). Ce paramètre est d'autant plus important que "les racines du transnationalisme sont impériales, plutôt que postcoloniales ", si l'on en croit Kevin Grant, Philippa Levine et Frank Trentmann ${ }^{13}$, qu'il s'agisse des résistances transnationales, des instruments politiques conçus pour les 
contrer ou des projets alternatifs d'une citoyenneté impériale qui irait au-delà de l'Etatnation. L'idée de Commonwealth ne peut se dissocier, de fait, des débats sur une fédération et une citoyenneté impériales au tournant du XXe siècle qu'ont étudiés David McIntyre et Daniel Gorman - et Frederick Cooper s'est récemment intéressé à ces questions de citoyenneté alternative, conçue hors du prisme de l'Etat-nation dans les négociations de la fin de l'empire français, suggérant de fait de nouvelles pistes de recherche sur les cosmopolitismes après $1945^{14}$. Si l'étude des transnationalismes intellectuels et culturels a permis de nouvelles lectures ${ }^{15}$, le rôle des diasporas dans l'élaboration de nouvelles relations internationales reste cependant encore peu étudié, notamment au sein du Commonwealth.

L'évolution des travaux de l'historien John Darwin est également révélateur de la mise en perspective du Commonwealth des Nations dans un cadre global qui le dépasse à la fois chronologiquement et spatialement. Là où les premiers travaux de John Darwin étaient avant tout centrés sur la fin de l'empire britannique, After Tamerlane: The Rise and Fall of Global Empires, 1400-2000 (2007) et Unfinished Empire: The Global Rise of Britain (2012) ne consacrent qu'une quarantaine et une soixante de pages (sur plus de 400 et 500 pages respectivement) à la grande période des indépendances politiques et à ses suites. Pour Darwin, la variation des cadres géopolitiques et géoculturels d'analyse dans la longue durée permet d'éviter une histoire européenne linéaire, enfermée dans le prisme de "l'occident ", tout en s'interrogeant sur ce qui distinguerait, "qualitativement», les empires européens des autres types d'empire. Dans les deux cas, l'objectif est bien de questionner, préciser, nuancer les contours géographiques, conceptuels, civilisationnels de «l'Europe ». C'est également ainsi que l'empire comme " projet » peut être compris : « inachevé, désordonné, une masse de contradictions, d'aspirations et d'anomalies ${ }^{16}$ ». Il rejoint ici Frederick Cooper et Ann-Laura Stoler, pour qui la recherche en sciences humaines, et particulièrement celle qui porte sur l'histoire des empires, ne doit pas perdre de vue les contingences que la longue durée et le comparatisme permettent de mettre au jour ${ }^{17}$.

Ces travaux sont très précieux pour l'élaboration d'une histoire du Commonwealth distanciée des premiers écrits articulés autour de la transformation de l'empire en Commonwealth - qu'il s'agisse, parmi les acteurs politiques comme parmi les historiens, de présenter une décolonisation britannique plus "pacifique» que celle de ses voisins européens ou de critiquer la poursuite de pratiques impériales et coloniales bien au-delà des indépendances politiques. Les nouvelles études sur l'usage de la force, par l'armée et la police, en contexte coloniaux et métropolitains, ou sur l'influence des expériences coloniales du maintien de l'ordre et des pratiques de détention sur les politiques de sécurité nationale et d'interventions extérieures par le Royaume-Uni post-colonial s'appuient sur une étude précise des pratiques administratives et de la législation ${ }^{18}$. De même, les travaux d'Elizabeth Buettner sur «l'Europe après l'empire » s'appuient sur les approches démographiques et sociales qui lui avaient déjà permis de mettre au jour les circuits familiaux qui, tout à la fois matériels, émotionnels et intellectuels, reliaient la Grande-Bretagne à son empire indien ${ }^{19}$. La place nouvelle accordée aux guerres d'empire dans l'histoire des décolonisations ${ }^{20}$ et aux guerres de mémoire dans les sociétés postcoloniales suggère qu'il est de toute manière difficile, voire impossible, d'éluder la question de l'impact des décolonisations sur les sociétés contemporaines.

Les travaux récents sur le Commonwealth ont pris acte de ces évolutions. En 2013, la thèse d'Usha Iyer, fondée sur les archives de l'Imperial Cricket Conference et du 
Marylebone Cricket Club entre 1947 et 1965, proposait une histoire transnationale du Commonwealth $^{21}$. Si la tenue des Jeux du Commonwealth à Glasgow en 2014 a suscité plusieurs doctorats sur les relations entre sport et identités nationales (notamment sur l'insertion de l'Ecosse dans les réseaux britanniques, Commonwealth et internationaux alors que le referendum sur l'indépendance avait lieu la même année ${ }^{22}$ ), les Jeux ont également donné lieu à des travaux en sociologie, notamment dans le domaine des études urbaines, et en médecine. Centrée sur la politique, la gestion et l'évaluation en matière de santé, la thèse d'Anna Cunningham sur le volontariat chez les jeunes montrait que les Jeux ont creusé les inégalités car ceux qui étaient le plus à même de participer étaient aussi ceux qui avaient le moins besoin des opportunités offertes en termes de santé physique et mentale ${ }^{23}$. De même, Maureen Kidd mettait en garde quant à l'impact réel que des évènements comme les Jeux pouvaient avoir sur le changement social, à partir de l'enquête sociologique menée dans les quartiers est de Glasgow, au cœur des dispositifs sportifs ${ }^{24}$. Certes, il semble que le Commonwealth soit avant tout un cas d'étude pertinent dans la conjoncture politique en Ecosse (l'Université de Glasgow étant très représentée), plutôt qu'un objet d'intérêt universitaire en soi. Mais ceci n'enlève rien au fait que ces études offrent aux chercheurs du champ Commonwealth un renouveau de leurs perspectives (précisément peut-être en raison de leur plus grande distance vis-à-vis de l'organisation). Les nouvelles perspectives des dernières années s'ancrent également dans un renouvellement des sources. Un exemple particulièrement marquant est la thèse de Tom Wilson, actuellement responsable des collections et de la recherche au Design Museum à Londres, sur l'Imperial Institute, fondé à Londres en 1887, et le Commonwealth Institute, qui prit sa suite de 1962 à $1997^{25}$. Dans son étude diachronique des galeries d'exposition, Wilson examine la transition de l'empire au Commonwealth à l'aune de la présentation des objets, croisant études muséales et théorie critique postcoloniale, afin de comprendre comment (et dans quel but) les concepts d'Empire et de Commonwealth ont été présentés au public britannique. S'il s'intéresse aux objets en eux-mêmes, le travail de Wilson porte également sur les techniques d'exposition pour éclairer les vestiges et héritages des imaginaires impériaux dans le Commonwealth contemporain. Si l'histoire du Commonwealth, notamment dans une perspective britannique, est ancrée dans le (post)empire, c'est aussi le renouvellement des concepts et des sources de l'histoire impériale qui a stimulé de nouvelles écritures du Commonwealth. Tout aussi important, cependant, a été - et demeure - le croisement avec d'autres disciplines et champs d'études.

\section{Cultures et espaces diplomatiques : nouvelles géographies du Commonwealth}

14 La grande force du courant postcolonial, affirme Achille Mbembe, «ce n'est pas seulement la radicalité de son éclectisme. C'est surtout le fait que le courant postcolonial est parvenu à décentrer le questionnaire des humanités ${ }^{26}$ ». Le "postcolonial», comme c'est désormais bien connu, n'a pas tant une acception chronologique, qui marquerait un « après » les empires, qu'une acception épistémique, « au-delà » des empires et du prisme d'une histoire nationale. De ce point de vue, l'une des principales contributions de ce courant est, comme l'écrit Emmanuelle Sibeud, d'« insiste[r] sur la nécessité de conjurer des dispositifs idéologiques que la décolonisation aurait laissés pratiquement intacts et [de] donne[r] ainsi une actualité nouvelle à l'anticolonialisme ${ }^{27} \%$. Les études 
postcoloniales présentent, certes, un certain nombre de limites, dont il faut prendre acte. Le travail sur les représentations, les imaginaires et le savoir ne peut se faire qu'à travers "la reconstruction minutieuse des contextes économiques et politiques ${ }^{28}$ ", des «ressources matérielles, sociales et culturelles ${ }^{29}$ » des individus parce qu'aucune « catégorie », aucune « identité » n'existe en soi ou hors du temps ${ }^{30}$. Le défi, en réalité, est de proposer des recherches " pleinement postcoloniales, c'est à dire conscientes des biais imposés par l'héritage intellectuel et culturel de la colonisation sans en faire pour autant leur unique objet», défi aussi ambitieux que nécessaire, comme le pose si bien Emmanuelle Sibeud ${ }^{31}$.

En Grande-Bretagne, le Birmingham Centre for Contemporary Cultural Studies (BCCS), fondé en 1964, avait déjà joué un rôle fondamental dans la révision des études impériales et des sociologies des fins d'empire. La réflexion très stimulante de Bill Schwarz sur les temps décalés des décolonisations, avec une décolonisation métropolitaine plus tardive, trouve ses origines dans le travail du BCCS, en collaboration avec Stuart Hall et Paul Gilroy. La revue interdisciplinaire Race and Class, née en 1974, participe également de la constitution d'un Commonwealth à la croisée des études internationales, des études sociales, des études impériales et postcoloniales. Les « autres » Afriques, et notamment l'Afrique lusophone qui obtient alors l'indépendance, figurent également en très bonne place, donnant une nouvelle vigueur à la réflexion sur les conférences panafricaines et afro-asiatiques, et les sites multiples de racisme et d'oppression, que le premier éditorial de Race avait évoqué dans le tout autre contexte institutionnel de 1959. Certains des articles publiés dans la revue s'inscrivent également dans le courant des "Black studies ", souvent très militant - même si les engagements se marquent de façon moins radicale qu'aux Etats-Unis. Plus récemment, l'affirmation des «Black studies » au Royaume-Uni s'est également nourrie de la critique formulée au sein des études latino-américaines notamment par des chercheurs d'origine latino-américaine en poste aux Etats-Unis - à l'encontre des études postcoloniales. Si plusieurs chercheurs américains avaient déjà reproché aux « études postcoloniales » de ne s'intéresser qu'aux décolonisations récentes des empires coloniaux européens et de faire abstraction du cas des Etats-Unis, Walter Mignolo ou Ramón Grosfoguel estiment que la pratique des études postcoloniales et subalternes conduit trop souvent à « une critique eurocentrée de l'eurocentrisme », qui accorde une place excessive aux penseurs européens de la postmodernité et finit en réalité par produire "des études au sujet des subalternes plutôt que des études avec et depuis une perspective subalterne ${ }^{32}$ ». Si la spécificité même du "décolonial» reste à préciser et si l'approche suscite des critiques à travers les disciplines, elle peut aussi permettre de mieux évaluer l'influence du passé sur le présent et les relations de pouvoir, au sein des sociétés comme sur la scène internationale.

Ces préoccupations se retrouvent dans l'intérêt accru porté par la recherche récente à une histoire culturelle de la diplomatie et à une histoire des diplomaties culturelles. Le Commonwealth des Nations est, par ses héritages impériaux et coloniaux et par ses politiques contemporaines, un facteur structurant des relations internationales et exerce, dans certains cas, une influence directe sur les équilibres politiques et culturels nationaux. Le Commonwealth n'est donc pas, contrairement à ce que l'état des recherches peut parfois laisser penser, un acteur négligeable des relations internationales contemporaines. Ecrire l'histoire du Commonwealth, et de la Grande-Bretagne dans le monde, c'est donc aussi s'interroger sur les articulations entre culture et pratiques culturelles, et sur les éléments constitutifs d'une culture diplomatique. De ce point de 
vue, la géographie politique et culturelle a considérablement enrichi les approches de l'histoire du Commonwealth. Le souci d'historiciser les décisions, de distinguer le particulier du général et du systémique a aussi mené la recherche à «spatialiser » ces mêmes décisions. Comme y invitait le tournant spatial des sciences sociales, les recherches sur les métagéographies et les géographies culturelles ${ }^{33}$, la réflexion doit alors s'orienter dans deux directions: déterminer l'influence des lieux de décision sur la formulation et le contenu même des politiques décidées et, d'autre part, évaluer dans quelle mesure ces décisions produisent à leur tour de nouveaux espaces de savoirs et de pratiques. Parallèlement, la critique des métagéographies a incité à une relecture des évènements et périodes historiques.

17 Avec les débats - politiques et universitaires - sur les mémoires et commémorations des empires et fins d'empire, l'histoire du Commonwealth a pris une certaine matérialité dans le Londres contemporain. CEuvres du sculpteur Ian Walters, les bustes de Nelson Mandela à l'extérieur du Royal Festival Hall, de Trevor Huddleston et d'oliver Tambo devant South Africa House, ne sont pas de simples symboles de la lutte pour l'émancipation en Afrique du Sud, du Mouvement Anti-Apartheid et de l'African National Congress. L'histoire de leur conception, localisation et transformation participe à la fois de la sociologie des mouvements racistes et antiracistes et de la pratique de la ville. Plus récemment, l'inauguration des statues de Nelson Mandela (2007) et du Mahatma Gandhi (2015) à Londres participe à la matérialisation des influences africaines et indiennes sur le centre du pouvoir britannique, tout comme les plaques bleues d'English Heritage marquant les résidences de Jawarharlal Nehru à Notting Hill (1910-1912), Jomo Kenyatta à Pimlico (1933-1937) ou encore Ruth First et Joe Slovo à Camden (1966-1978) montrent les circulations qui ont fait l'empire, le Commonwealth et le Royaume-Uni contemporain. Parallèlement, le choix récent de Londres et de Windsor pour le Sommet des Chefs d'Etat et de Gouvernement du Commonwealth au printemps 2018 - aux dépens du nord de l'Angleterre et des Midlands qui avaient été un temps pressentis - suggère que la définition et la pratique des lieux de pouvoir demeurent un objet pertinent et important de l'étude des mécanismes diplomatiques et sociétaux du Commonwealth actuel.

Les récents travaux des géographes ont renouvelé l'histoire du Commonwealth à trois égards au moins. Premièrement, s'il est pris acte de la critique formulée par l'école indienne des «études subalternes » autour de Ranajit Guha à partir de la fin des années 1970, face à la violence épistémologique des sciences humaines et sociales largement dominées par les pensées et les espaces européens et occidentaux ${ }^{34}$, le concept de " subalternité » a aussi été revisité. Certains géographes ont prolongé la réflexion de Mohammed Ayoob sur le "réalisme subalterne », critique du prisme euro-centré de la théorie des relations internationales ${ }^{35}$, en proposant le concept de "géographie subalterne » qui, contrairement à l'anti-géopolitique, reste centré sur les Etats et les institutions qui en dérivent et adoptent l'acception militaire de "subalterne ", ni tout à fait au cœur des prises de décision ni tout à fait en dehors des structures ${ }^{36}$. Les ambiguités, plus que l'exclusion tranchée, des positions subalternes permettent ainsi de mieux évaluer les imaginaires, pratiques et résultats des nouveaux diplomates dans des institutions telles que le Secrétariat du Commonwealth après 1965, à la croisée entre héritages coloniaux et créativité postcoloniale, comme l'a montré Ruth Craggs ${ }^{37}$. Cela rejoint également l'utilisation par Fiona McConnell de la notion de liminarité en anthropologie culturelle pour comprendre le changement dans les ordres mondiaux institutionnalisés ${ }^{38}$. Deuxièmement, les géographes se sont intéressés à la diplomatie 
comme spectacle ou performance, à la suite de l'analyse proposée par Naoko Shimazu de l'importance, avant tout symbolique, de la Conférence de Bandoeng, dans le cadre d'une géographie politique qui souligne l'impact de l'environnement sur les pratiques et les cultures diplomatiques ${ }^{39}$. Dans son étude des voyages travaillistes en Afrique, notamment lors de l'année du " vent du changement », l'historien Nicholas Owen emprunte à la fois à l'histoire, à la géographie et à la littérature pour élargir le cadre spatial du Royaume-Uni mais aussi pour l'affiner, en s'intéressant très précisément aux expériences personnelles pour tenter de mieux définir, nuancer ou remettre en question une «spécificité » de la sphère Commonwealth ${ }^{40}$. Enfin, les approches géographiques de la diplomatie ont (ré)activé plusieurs notions à démêler pour comprendre décisions et politiques, personnelles et collectives - notamment l'hospitalité, la créativité, l'émotion et l'aspiration, et leur impact sur la géopolitique. Comme le rappelait récemment Stuart Ward lors du colloque d'avril 2018 à Cambridge sur "The Commonwealth Effect ", le Commonwealth constitue un réseau de gouvernance globale inédit, mais où «l'affect » rend parfois l'évaluation de son " effet» difficile à quantifier. Ces considérations sont au cœur des études de Ruth Craggs sur le rôle des hôtels en Rhodésie du Sud comme espaces constitutifs d'une politique locale, nationale et internationale à l'ère de la mobilisation contre la ségrégation ${ }^{41}$, de Josiah Brownell sur les liens entre l'espace de Rhodesia House à Londres et les structures constitutionnelles ${ }^{42}$, et de JoAnn McGregor sur l'expérience de l'exil chez les étudiants zimbabwéens présents à Londres pendant la guerre de libération, et son impact sur les relations avec la diversité des réseaux militants contemporains ${ }^{43}$. Pour les historiens du Royaume-Uni et de l'Afrique, ces études suggèrent également des voies pour mieux connecter (ou pour mieux comprendre les connections partielles ou inexistantes), entre l'histoire de l'anti-apartheid, l'histoire du cosmopolitisme urbain et l'histoire du racisme / anti-racisme dans les années 1960 et $1970^{44}$.

\section{Globalisation, langue(s) et culture(s)}

19 L'étude du Commonwealth, comme communauté de pratiques et comme organisation internationale, amène également à s'interroger sur les processus de globalisation, parfois masqués par la fragmentation des grands ensembles en Etats-nations à l'ère des indépendances. Pour Martin Thomas et Andrew Thompson, "les processus de globalisation de la période post-1945 trouvent leurs origines dans les conflits armés de la décolonisation", à la fois parce que ces conflits furent une cause, plus qu'un simple "symptôme" des bouleversements dans les relations internationales et parce que les méthodes et pratiques de contestation "définirent de nouveaux critères normatifs de violence antiétatique par des acteurs et groupes non-étatiques ${ }^{45}$ ». La décolonisation fut aussi globalisation, dans le sens où elle a produit des tendances normatives dominantes, $\mathrm{y}$ compris dans les organisations internationales et les diplomaties multilatérales. Le transnationalisme, comme l'ont rappelé à plusieurs reprises Pierre-Yves Saulnier ou Arika Iriye, n'est pas en soi progressiste ou libérateur, et certainement pas a-normatif. L'histoire du développement, de l'humanitaire et des corporations transnationales montre, au-delà de l'inachèvement de la décolonisation, les racines impériales, spatiales et culturelles, des acteurs transnationaux contemporains. Plusieurs de ces groupes étant engagés d'abord simultanément au sein de l'empire britannique et dans les réseaux du Commonwealth, l'histoire de l'humanitaire à la fin des empires permet de mieux comprendre les fondements idéologiques et pratiques, impériaux et internationaux, de 
certains des acteurs qui font aujourd'hui le Commonwealth « des peuples », non officiel, qui a souvent revendiqué un rôle de promoteur des «droits» face au Commonwealth intergouvernemental. A la suite des travaux de Stuard Ward et Alex May sur les célébrations du Commonwealth au Royaume-Uni, les travaux d'Emily Baughan sur Save the Children ou d'Anna Bocking-Welch sur les campagnes d'éradication de la faim dans le monde ou du Commonwealth Day permettent de mieux comprendre la place du Commonwealth dans la société britannique et ses imaginaires. Si l'utilisation du «Commonwealth» dans la campagne de 2016 sur l'Union européenne et, surtout, au lendemain du vote en faveur du "Brexit", amènera certainement un regain d'intérêt pour ces questions, le champ reste pour l'instant assez peu exploré46.

Face à une histoire globale inscrite initialement dans une interprétation économique de la mondialisation vue comme progrès, Paul Gilroy avait préféré au "global» le " planétaire ", qui « suggère à la fois la contingence et le mouvement ${ }^{47}$ ». La globalisation peut aussi être identifiée comme une " anglobalisation ${ }^{48}$ ", qui accompagne et alimente en partie les récits positifs des empires comme mode d'organisation du monde international, militant parfois pour un retour à une certaine Pax Britannica ${ }^{49}$. En ce sens, l'histoire du Commonwealth ne peut se dissocier de l'émergence d'une " "communauté épistémique" des experts travaill[ant] en langue anglaise ${ }^{50}$ ». Pour Amitav Banerji, ancien directeur des affaires politiques au Secrétariat du Commonwealth, les capacités d'action du Commonwealth dérivent en grande partie de l'héritage colonial commun des pays membres, défini autour de la langue anglaise et de traditions parlementaires, juridiques et éducatives partagées - ce qui milite pour une ouverture exceptionnelle, au cas par cas, à des pays qui ne répondent pas à ces caractéristiques ${ }^{51}$. Comme l'indiquait Jean-Claude Redonnet, «si le Commonwealth n'avait eu que la langue anglaise à partager, il n'aurait probablement pas survécu aux bouleversements de la décolonisation ${ }^{52}$ ». Ceci ne signifie en rien que l'anglais ne joue pas un rôle important dans les activités de l'organisation, ni qu'il ne faille ignorer qu'il est initialement la langue de la puissance coloniale et actuellement celle (aussi) de la puissance américaine. Toutefois, non seulement l'anglais lui-même comporte de multiples variantes, mais sa place même au sein des pays membres du Commonwealth et au sein du Commonwealth lui-même est mise en perspective, parfois décentrée, parfois réappropriée.

21 En études anglophones, où des travaux majeurs sur les questions postcoloniales ont été publiés dès les années $1980^{53}$, il existe indéniablement une tension constante entre la volonté d'aller au-delà des aires britanniques et nord-américaines et l'impossibilité de faire du monde anglophone une "aire", au risque de vouloir trop embrasser de façon impérialiste, ou de reproduire des divisions « coloniales » trop rigides et simplificatrices. En ce qui concerne l'Afrique - et la même observation s'applique tout aussi bien à d'autres régions de ces mondes « anglophones » dont l'Asie du Sud et du Sud-Est - Achille Mbembe souligne à juste titre que l'analyse peut facilement être « emportée par le délire verbal, les slogans et l'indigence linguistique (les uns ne lisent que le français, les autres ne lisent que l'anglais, bien peu parlent les langues locales $)^{54} »$. Dans ces conditions, le dialogue avec les réseaux d'autres études (africaines, asiatiques ...), fondés sur l'interdisciplinarité, est indispensable pour parer, au moins en partie, à ces risques. Si la réflexion permanente sur la langue et la culture qui accompagne l'approche des études anglophones peut en faire une courroie de transmission (et de production) précieuse dans le dialogue entre les vastes champs des écrits universitaires anglophones et francophones, la rare maîtrise de langues non-européennes continue d'être un obstacle à l'appréhension des mondes 
africains, caribéens, indiens/asiatiques et pacifiques du Commonwealth des Nations militant ainsi pour un dialogue suivi avec les spécialistes des différentes aires du Commonwealth. Au Cameroun, les universitaires qui se sont le plus intéressés au Commonwealth depuis l'entrée du pays au sein de l'organisation en 1995 sont aussi ceux qui travaillent sur les langues minoritaires. Les travaux de Kizitus Mpoche sur l'apprentissage du bilinguisme au Cameroun et sur la langue limbum, parlée dans les Grassfields de l'ouest du pays ${ }^{55}$ s'inscrivent aussi dans l'importance qu'il accorde à la coopération interuniversitaire au sein du Commonwealth. A l'Université de Yaoundé I, le fait que le nombre d'étudiants inscrits dans les cours de littérature du Commonwealth a quasiment quintuplé depuis le milieu des années $1990^{56}$ ne s'explique pas simplement par un intérêt accru pour le postcolonial, mais aussi pour une langue qui, si elle est langue officielle aux côtés du français, est en réalité minoritaire. A cet égard, la référence explicite au Commonwealth dans l'intitulé des modules n'est sans doute pas fortuite. L'admission du Cameroun en 1995, ainsi que celle du Rwanda en 2009, incite à reconsidérer l'importance des usages pratiques, politiques et symboliques d'une langue en politique étrangère, et de l'absence du recours à la traduction ou à l'interprétation dans les transactions au sein du Commonwealth.

L'histoire du Commonwealth s'est aussi nourrie, si l'on considère la sphère africaine, des travaux d'Ali Mazrui sur les relations entre anglais et swahili en Afrique de l'Est (1977), ou encore des écrits critiques et littéraires de Ngugi wa Thiongo sur la décolonisation des esprits (1986). Si certaines œuvres sont parfois citées dans le contexte d'études sur les fins d'empire, il ne s'agit pas simplement d'illustrer tel ou tel propos mais bien de montrer la manière dont les conditions de création, de production et de diffusion - y compris les connections locales et internationales des éditeurs - créent une certaine culture, politique et culturelle ${ }^{57}$. Dans son étude sur la culture littéraire au Ghana colonial publiée en 2002, Stephanie Newell démontre les liens entre esthétique littéraire et histoire coloniale à partir de nombreux fonds d'archives, ouvrant de nouvelles perspectives sur des figures du nationalisme ouest-africain importantes comme J.E. Casely Hayford, J.B. Danquah ou I.T. Wallace-Johnson ${ }^{58}$. Si la dimension culturelle du Commonwealth n'a pas la visibilité - ni les programmes et les financements - de la Francophonie, elle opère néanmoins à travers de multiples réseaux, souvent non-gouvernementaux mais en correspondance directe avec le Commonwealth officiel, qui méritent eux aussi d'être inscrits dans une histoire de l'organisation.

Lors de la réunion d'inauguration du Centre for Postcolonial Studies au sein de l'Institute of Commonwealth Studies à Londres en janvier 2016, la très grande majorité des directeurs de centres d'études postcoloniales au Royaume-Uni insistaient sur l'importance d'approches intégrant les composantes culturelles, littéraires et linguistiques des objets étudiés. Les chercheurs actuellement en poste dans ces centres partagent de fait les critiques parfois formulées à l'encontre des «cultural studies » notamment un éloignement des approches historiques et sociologiques vers une sacralisation des objets et textes indépendamment de leur contexte ${ }^{59}$ - et qui ont pu aussi s'appliquer à certaines approches des "postcolonial studies». Mais ils formulent également des critiques à l'encontre d'une histoire ou d'une sociologie qui ferait abstraction des conditions linguistiques et littéraires au sens large de leurs objets. L'histoire de la discipline, comme le rappelait récemment Elleke Boehmer, doit s'intéresser aux relations entre littérature postcoloniale et écriture de l'histoire coloniale et postcoloniale, ainsi qu'à la théorie et à l'usage des termes « monde », « postcolonial » et 
"Commonwealth»- et ceci vaut pour la littérature comme pour les différents champs des sciences sociales. Ceci est fondé sur la volonté d'aller «au-delà de certains discours triomphalistes d'une uniformité anglophone globalisée afin de mieux comprendre la complexité et la diversité - linguistique, culturelle et politique - du monde dans lequel nous vivons ${ }^{60} »$. Or, c'est aussi à cela, précisément, que s'attachent les études anglophones (même si elles sont aussi le véhicule de l'apprentissage et de la diffusion de la langue anglaise). Il ne s'agit absolument pas de négliger ou de subsumer les spécificités propres à chaque discipline mais plutôt de mieux prendre en compte la manière dont, par exemple, l'écriture fictionnelle et la réception des œuvres ont pu jouer un rôle dans la formulation de politiques internationales et dans la médiatisation et la mémorialisation des conflits coloniaux et post-coloniaux dans les sociétés.

\section{Le Commonwealth comme catégorie d'analyse : apports et limites}

Pour conclure que le Commonwealth est un prisme d'analyse pertinent pour l'étude des sociétés des pays membres, encore faut-il pouvoir mesurer sa place parmi les autres organisations, structures ou personnes considérées. Dans un certain nombre de cas, le Commonwealth comme catégorie d'analyse demeure pertinent parce qu'il correspond à des réseaux d'activités bien réels ou à des traditions partagées. Un exemple récent en est le numéro spécial coordonné par Sue Onslow sur la liberté des médias dans le Commonwealth ${ }^{61}$. Il ne s'agit donc pas d'enfermer une recherche dans un prisme hérité du colonialisme mais bien d'utiliser ce prisme pour aller au-delà. Les sciences politiques, de fait, de façon peut-être plus tranchée que l'histoire, ont accordé une place grandissante à la méthode comparatiste dans les études sur le Commonwealth. La revue Comparative and Commonwealth Politics a d'abord paru sous le titre The Journal of Commonwealth Political Studies en 1961, avant de devenir The Journal of Commonwealth and Comparative Politics en 1998, puis d'adopter son titre actuel. Mais dès le premier numéro, l'éditorial faisait du comparatisme et de l'interdisciplinarité dans les sciences humaines et sociales un de ses piliers. Encourageant juristes, économistes, historiens et anthropologues à dialoguer avec les politologues, la revue décrivait son objet premier comme étant «le Commonwealth en tant qu'institution, les relations entre ses membres, et les politiques et gouvernements des pays du Commonwealth ${ }^{62} »$. Le tout premier article, de fait, s'intitulait "Westminster's export models: the legal framework of responsible government ${ }^{63} »$. Ces dernières années, la réflexion sur ces questions s'est à la fois prolongée et renouvelée, comme en témoignent les travaux de Harshan Kumarasingham, chercheur néo-zélandais dont les racines sont en partie en Asie du Sud, sur les «Eastminsters », les modèles politiques indiens et sri-lankais, adaptés du système de Westminster, croisant influences extérieures et intérieures, globales et locales, sur les institutions politiques, leurs principes et leurs cadres d'application. Fondant ses travaux sur une solide sociologie politique historique comparée, Kumarasingham montre ainsi que la recherche sur les transferts ne conduit pas nécessairement à renforcer l'ancrage national des historiographies, à condition de donner toute son importance au croisement comme lieu de production ${ }^{64}$.

Une des difficultés à laquelle sont confrontés les historiens du Commonwealth - comme ceux des empires - est de déterminer quelle place l'organisation occupe réellement parmi les populations des pays membres. Si pour Bernard Porter, l'empire a eu tout au plus une 
influence marginale et n'a surtout intéressé que quelques éléments de la société britannique - essentiellement les élites et les décideurs directement impliqués dans les affaires coloniales - ses principaux opposants, dont John MacKenzie et Stuart Ward, soulignent néanmoins les ambiguïtés, les zones d'ombre, les indifférences attestées des Britanniques aux questions d'empire et de décolonisation. L'histoire du Commonwealth n'est pas circonscrite par l'ouverture progressive des archives des Etats, associations et individus - même si elle en bénéficie bien sûr largement. Elle est aussi cette histoire du temps présent, définie par Robert Frank à la suite de Paul Ricoeur comme « l'histoire d'un passé récent comportant un "point de clôture" [...] même si les effets de mémoire font qu'il n'est pas révolu », et « une histoire du temps présent non clos et dont on ne connait pas le mot de la fin ${ }^{65}$ ». Les soixante "Commonwealth Oral Histories», recueillies par Philip Murphy, Sue Onslow et Ruth Craggs, actuellement en cours de mise en ligne, représentent une nouvelle ressource extrêmement riche sur l'histoire du Commonwealth depuis $1965^{66}$.

Si l'on considère les thèses récemment soutenues au Royaume-Uni et où le Commonwealth figure comme périmètre géographique et conceptuel, deux constats émergent. D'une part, les études de droit - qu'il s'agisse de l'anticorruption, des droits LGBT ou du rôle des juges en common law ${ }^{67}$-occupent une place aussi importante que l'histoire. D'autre part, si le Royaume-Uni et les dominions priment dans les travaux sur le Commonwealth d'avant 1965, c'est avant tout en relation aux Caraïbes que le Commonwealth contemporain est pensé comme une catégorie pertinente et, dans une moindre mesure, en Afrique. Au-delà des thèses, de fait, c'est aussi dans les Caraïbes que le facteur Commonwealth revient fréquemment dans les études en économie, sur le développement international, par exemple, ou sur le commerce. Mais si le prisme "Commonwealth" n'est que très rarement utilisé de façon exclusive ${ }^{68}$, force est néanmoins de constater que davantage de travaux mériteraient d'être conduits sur la manière dont il est utilisé dans l'appréhension des scènes nationales et internationales, en concurrence ou en complémentarité avec d'autres. Si l'on prend l'exemple de l'Afrique, l'histoire des décolonisations a bien souvent divisé le continent - entre l'Afrique $\mathrm{du}$ Nord et l'Afrique sub-saharienne, ou les Afriques diversement appelées "francophones", "lusophones" et "anglophones", et recouvrant en réalité des héritages politiques, culturels et linguistiques qui ne peuvent s'accommoder ni des déterminants «linguistiques", ni des anciennes frontières impériales (qui ne les recoupent qu'en partie, notamment dans le cas « francophone»). Plus que les historiens des relations internationales, ce sont souvent les spécialistes de philosophie, d'histoire des idées et d'études postcoloniales qui ont réfléchi aux connexions directes, notamment dans la période des indépendances - contrairement aux débats actuels sur l'impact des printemps arabes sur l'Afrique et les migrations, et aux recherches sur les échanges transsahariens aux périodes précoloniale et coloniale ${ }^{69}$.

\section{Conclusion}

En 2014, la commission de la Chambre des lords sur le «soft power» accordait au Commonwealth une importance clé, notamment en raison de ses « réseaux informels et personnels " multiformes, qui permettent de reconsidérer les moyens de l'influence (britannique et autre) dans le monde. En conséquence, elle appelait à ce que le Commonwealth soit réellement pris en considération, pas uniquement par le Foreign and 
Commonwealth office, mais par l'ensemble des ministères concernés par les questions de défense et de sécurité, de commerce et de développement, d'éducation et d'environnement ${ }^{70}$. Mais l'intérêt en apparence renouvelé que la campagne de 2016, et surtout le vote en faveur du «Brexit », ont donné au Commonwealth, est loin d'être sans danger : la rhétorique et les projets politiques (particulièrement parmi les partisans du «Brexit») semblent parfois en décalage avec la réalité de l'influence britannique, les intérêts propres aux autres puissances de l'organisation et l'importance (toute relative) que la population britannique elle-même semble réellement lui porter. Comme le soulignait récemment Philip Murphy, le Commonwealth fait ainsi face à deux écueils au moins : celui de devenir l'incarnation d'un « empire 2.0 » et celui de se dissoudre dans un idéalisme impuissant ${ }^{71}$. Ces risques sont bien réels, mais ne résument cependant pas à eux seuls le Commonwealth. Pour la recherche, le défi est de maintenir l'équilibre entre le Commonwealth comme idée, comme pratique et comme institution (trois aspects qui évoluent dans le temps et que différents acteurs formulent, mettent en œuvre et interprètent différemment), afin de proposer une étude des relations internationales dans toute leur épaisseur. C'est ainsi que le Commonwealth en tant qu'organisation a toute sa place dans l'étude et la pratique des ordres et désordres internationaux. Et c'est en ce sens aussi que l'histoire de l'Etat et de la société britanniques aux XXe et XXI siècles peut parvenir à s'émanciper du cadre national - sans pour autant l'ignorer. Pour l'histoire du Commonwealth écrite depuis le champ des études anglophones, ou des études britanniques, la nécessité d'un dialogue avec les spécialistes d'autres aires, représentées au sein du Commonwealth et en dehors (comme les Etats-Unis ou l'Amérique latine) est une exigence tout aussi importante que le dialogue interdisciplinaire. Comme le soulignait Sandra Pepera, membre de la division des affaires politiques au Secrétariat du Commonwealth avant de rejoindre le Ministère du développement international britannique puis d'occuper son poste actuel au National Democratic Institute à Washington, les institutions du Commonwealth ne se sont pas adaptées au changement générationnel au sein du Commonwealth, où « il n'y a pas deux leaders désormais - sans doute - qui puissent se référer à quelque sorte de passé commun ${ }^{72}$ » (contrairement aux premières générations où nombreux avaient été ceux qui d'Asie, d'Afrique et des Caraïbes étaient venus étudier à Londres). Cette évaluation du rapport à un "passé commun » à travers les générations et en relation avec d'autres réseaux d'action et de solidarité sera d'autant plus précise qu'elle interrogera les liens entre politique, culture et langues, dans les aspects matériels et intangibles, depuis les multiples points d'ancrage du Commonwealth des Nations contemporains. Si les débats autour du «Brexit» ont souligné l'intérêt d'une réévaluation de la place du Commonwealth dans la politique et les imaginaires britanniques, ils taisent en revanche ce que ce même Commonwealth représente pour le reste de l'organisation, de la Malaysie à la Barbade et à la Tanzanie, dans toute sa diversité, éclairant à nouveau les tensions entre échelles nationales, régionales et internationales dans l'étude des multilatéralismes et transnationalismes. C'est donc aussi en cartographiant les lieux de production de la recherche sur le Commonwealth (une vaste entreprise), en parallèle de ses lieux d'action et de mémoire que l'histoire du Commonwealth contemporain pourra continuer à se renouveler.

Mélanie Torrent est maître de conférences à l'Université Paris Diderot, et membre junior de l'Institut universitaire de France (2016). Ses recherches portent sur l'histoire connectée des fins d'empire en Afrique et sur la politique étrangère et la diplomatie britanniques contemporaines. 


\section{BIBLIOGRAPHIE}

AKO, Edward O., « From Commonwealth to Postcolonial Literature », CLCWeb : Comparative Literature and Culture, vol. 6, $n^{\circ} 2,2004$.

An., « Editorial », The Journal of Commonwealth Political Studies, vol.1, n¹, 1961.

ANYAOKU, Emeka, « The Modern Commonwealth », The Round Table, The Commonwealth Journal of International Affairs, vol. 100, n416, 2011.

ASHTON, Stephen R., « British Government Perspectives on the Commonwealth, 1964-71: An Asset or a Liability? ", Journal of Imperial and Commonwealth History, vol. 35, n¹, 2007.

АҮоОВ, Mohammed, «Inequality and theorizing in international relations : the case for subaltern realism », International Studies Review, vol. 4, n³, 2002.

BADIE, Bertrand, Nous ne sommes plus seuls au monde : un autre regard sur l' " ordre international ", Paris, La Découverte, 2016.

BARDOLPH, Jacqueline et al., Oppression et expression dans la littérature et le cinéma: Afrique, Amérique, Asie. Paris, L'Harmattan, 1981.

BARNETT, Michael, « Radical chic? Subaltern realism : a rejoinder », International Studies Review, vol. $4, n^{\circ} 3$.

BELL, Emma, « Normalising the Exceptional: British Colonial Policing Cultures Come Home », Mémoire(s), identité(s), marginalité(s) dans le monde occidental contemporain [En ligne], 10, document 9, 2013, http://mimmoc.revues.org/1286.

BROWNELL, Josuah, « "A sordid tussle on the Strand”: Rhodesia House during the UDI rebellion (1965-80) », Journal of Imperial and Commonwealth History, vol. 38, 2010.

BUETTNER, Elizabeth, Empire Families. Britons and Late Imperial India. Oxford, Oxford University Press, 2004.

BUETTNER, Elizabeth, Europe after Empire. Decolonization, Society and Culture. Cambridge, Cambridge University Press, 2016.

CHAKRABARTY, Dipesh, Provincializing Europe: Postcolonial Thought and Historical Difference, Princeton, Princeton University Press, 2000.

CHATTERJEe, Partha, The Black Hole of Empire: History of a Global Practice of Power., Princeton, Princeton University Press, 2012.

COOPER, Frederick, Colonialism in Question. Theory, Knowledge, History, Berkeley, University of California Press, 2005.

COOPER, Frederick, Citizenship Between Empire and Nation: Remaking France and French Africa, 1945-1960, Princeton, Princeton University Press, 2014.

COOPER, Frederick et STOLER, Ann-Laura, « Between Metropole and Colony: Rethinking a Research Agenda » in Frederick COOPER et Ann-Laura STOLER (ed), Tensions of Empire: Colonial Cultures in a Bourgeois World, Berkeley, University of California Press, 1997. 
CRAGGS, Ruth, « Towards a political geography of hotels : Southern Rhodesia, 1958-1962 », Political Geography, vol. 31, n, 2012.

CRAGGS, Ruth, « Subaltern geopolitics and the post-colonial Commonwealth, 1965-1990 », Political Geography, n65, 2018.

DARWIN, John (2007). After Tamerlane. The Rise \& Fall of Global Empires, 1400-2000. Londres, Allen Lane. DARWIN, John, The Empire Project, The Rise and Fall of the British World-System, 1830-1970, Cambridge, Cambridge University Press, 2009.

DARWIN, John, Unfinished Empire. the Global Expansion of Britain, Londres, Allen Lane, 2012.

DE SMITH, S.A., « Westminster's export models: the legal framework of responsible government », The Journal of Commonwealth Political Studies, vol. 1, n¹, 1961.

DOUKI, Caroline et MINARD, Philippe, « Histoire globale, histoires connectées : un changement d'échelle historiographique? ", Revue d'histoire moderne et contemporaine, vol. 54-4bis, n5, 2005. FERGUSON, Niall, Empire: How Britain Made the Modern World, Londres, Allen Lane, 2003.

FORSDICK, Charles et MURPHY, David, «Introduction: the Case for Francophone Postcolonial Studies » in Charles FORSDICK et David MURPHY (ed), Francophone Postcolonial Studies. A Critical Introduction, Londres, Arnold, 2003.

FRANK, Robert, « Une histoire problématique : une histoire du temps présent », Vingtième Siècle. Revue d'histoire, vol. 71, 2001.

GILROY, Paul, After Empire. Melancholia or Convivial Culture? Londres, Routledge, 2004.

GORMAN, Daniel, Imperial Citizenship. Empire and the Question of Belonging. Manchester, Manchester University Press, 2006

GRANT, Kevin, LEVINE, Philippa et TRENTMANN, Frank, Beyond Sovereignty: Britain, Empire and Transnationalism, c. 1880-1950, Londres, Palgrave Macmillan, 2007.

GRoSfoguel, Ramón, « Decolonizing Post-Colonial Studies and Paradigms of Political-Economy: Transmodernity, Decolonial Thinking and Global Coloniality », Transmodernity: Journal of Peripheral Cultural Production of the Luso-Hispanic World, vol. 1, n¹, 2011.

GROSSER, Pierre, « L'histoire mondiale/globale, une jeunesse exubérante mais difficile », Vingtième Siècle, vol. 110, n², 2011.

HALL, Ian, « The Revolt against the West: Decolonisation and its Repercussions in British International Thought, 1945-75 », International History Review, vol. 33, n²1, 2011.

HARROW, Kenneth et MPOCHE, Kitizus (dir.), Language, Literature and Education in Multicultural Societies: Collaborative Research on Africa. Newcastle, Cambridge Scholars Publishing, 2008.

KUMARAsingham, Harshan, A Political Legacy of the British Empire: Power and the Parliamentary System in Post-Colonial India and Sri Lanka, Londres, I.B. Tauris, 2013.

LEVINE, Philippa, « Gendering Decolonisation », Histoire@Politique, vol. 2, n¹1, 2010.

LEWIS, Martin W., WIGEN, Kären, The Myth of Continents. A Critique of Metageography, Berkeley, University of California Press, 1997.

LYDON, Ghislaine, On Trans-Saharan Trails. Islamic Law, Trade Networks and Cross-Cultural Exchange in Nineteenth-Century Western Africa, Cambridge, Cambridge University Press, 2009 
MANELA, Erez, The Wilsonian Moment. Self-Determination and the International Origins of Anticolonial Nationalism. Oxford, Oxford University Press, 2007.

MARKS, Shula, « History, the Nation and Empire: Sniping from the Periphery », History Workshop Journal, vol. 29, n¹, 1990.

MAZOWER, Mark, No Enchanted Palace: The End of Empire and the Ideological Origins of the United Nations. Princeton, Princeton University Press, 2009.

MAZOWER, Mark, Governing the World: The History of an Idea, 1815 to the Present. New York, London, Penguin, 2012.

MAZRUI, Ali, The Anglo-African Commonwealth: Political Friction and Cultural Fusion. Oxford, Pergamon, 1977.

MBEMBE, Achille, De la postcolonie, Paris, Karthala, 2000.

MBEMBE, Achille, « Qu'est-ce que la pensée postcoloniale ?», Esprit, vol. 12, 2006.

MCCONNELL, Fiona, «Liminal geopolitics : the subjectivity and spatiality of diplomacy at the margins ", Transactions of the Institute of British Geographers, vol. 41, n¹, 2017.

MCDOUGALL, James, SCHEELE, Judith, Saharan Frontiers. Space and Mobility in Northwest Africa, Bloomington, Indiana University Press, 2012.

MCGREGOR, JoAnn, « Locating exile: decolonization, anti-imperial spaces and Zimbabwean students in Britain, 1965-1980 », Journal of Historical Geography, vol. 57, 2017.

MCINTYRE, David W., The Britannic Vision. Historians and the Making of the British Commonwealth of Nations, 1907-1948, Basingstoke, Palgrave Macmillan, 2009.

MURPHY, Philip, The Empire's New Clothes: The Myth of the Commonwealth, Londres, Hurst, 2018.

NEWELL, Stephanie, Literary Culture in Colonial Ghana. 'How to play the game of life', Manchester, Manchester University Press, 2002.

NEWELL, Stephanie, « Afterword » in Derek R. PETERSON, Emma HUNTER et Stephanie NEWELL (dir.), African Print Cultures. Newspapers and their Publics in the Twentieth Century, Ann Arbor, University of Michigan Press, 2016.

onsLow, Sue (dir.), «The Commonwealth and Media Freedom », The Round Table: The Commonwealth Journal of International Affairs, vol. 107, $\mathrm{n}^{\circ} 2,2018$.

OWEN, Nicholas, « Four Straws in the Wind : Metropolitan Anti-Imperialism, January-February 1960 » in L.J. BUTLER et Sarah STOCKWELL (ed), The Wind of Change: Harold Macmillan and British Decolonization, Oxford, Oxford University Press, 2013.

REDONNET, Jean-Claude, Le Commonwealth. Politiques, coopération et développement anglophones, Paris, Presses universitaires de France, 1998.

RÉVAUGER, Jean-Paul, « La Grande-Bretagne de Tony Blair entre deux modèles de société », Observatoire de la société britannique, vol. 1, 2006.

ROCCHI, Jean-Paul et SYLVANISE, Frédéric, « Black Europe: Subject, Struggles and Shifting Perceptions », Palimpsest, vol. 4, n², 2015.

SCHILLER, Nina Glick, SALAZAR, Noel B., « Regimes of Mobility Across the Globe ", Journal of Ethnic and Migration Studies, vol. 39, $\mathrm{n}^{\circ} 2,2013$.

SCHWARZ, Bill, Memories of Empire. The White Man's World, Oxford, Oxford University Press, 2011. 
SHARP, Joanne, «Subaltern geopolitics : introduction », Geoforum, vol. 42, n³, 2011.

SHIMAZU, Naoko, «Places in Diplomacy », Political Geography, vol. 31, n6, 2012.

SHIMAZU, Naoko, « Diplomacy as theatre : staging the Bandung Conference of 1955 », Modern Asian Studies, vol. $48, \mathrm{n}^{\circ} 1,2014$

SIBEUD, Emmanuelle, « Post-colonial et Colonial Studies : enjeux et débats », Revue d'histoire moderne et contemporaine, vol. 51, $\mathrm{n}^{\circ} 4 \mathrm{bis}, 2004$.

SIBEUD, Emmanuelle, « Du postcolonialisme au questionnement postcolonial : pour un transfert critique », Revue d'histoire moderne et contemporaine, vol. 54, $n^{\circ} 4,2007$.

SINCLAIR, Georgina, At the End of the Line. Colonial Policing and the Imperial Endgame, 1945-1980,

Manchester, Manchester University Press, 2006.

SKINNER, Rob et LESTER, Alan, « Humanitarianism and Empire: New Research Agendas », The Journal of Imperial and Commonwealth History, vol. 40, n5, 2012.

THOMAS, Martin et THOMPSON, Andrew, « Empire and Globalisation: From 'High Imperialism' to Decolonisation ", International History Review, vol. 36, n 12014.

THOMPSON, Andrew (dir.), Britain's Experience of Empire in the Twentieth Century. Oxford, Oxford University Press, 2012.

VAN DAMME, Stéphane, «Comprendre les Cultural Studies : une approche d'histoire des savoirs ", Revue d'histoire moderne et contemporaine, vol. 51, n4 bis, 2004.

WA THIONGO, Ngugi, Decolonising the Mind: The Politics of Language in African Literature. Londres, James Currey, 1986.

\section{NOTES}

1. « an atypical and asymmetrical organisation », a " global exemplar of understanding and cooperation "; « important to look to the future of the Commonwealth, not through the prism of an imperial past, but as a new and growing organisation, able to deploy its unique attributes in global service"; ANYAOKU, Emeka, "The Modern Commonwealth», The Round Table, The Commonwealth Journal of International Affairs, vol. 100, $\mathrm{n}^{\circ} 416,2011$, p. 508, p. 500.

2. BADIE, Bertrand, Nous ne sommes plus seuls au monde: un autre regard sur l' "ordre international ", Paris, La Découverte, 2016, p. 108-109.

3. DOUKI, Caroline et MINARD, Philippe, «Histoire globale, histoires connectées : un changement d'échelle historiographique?", Revue d'histoire moderne et contemporaine, vol. 54-4bis, n5, 2005, p. 21.

4. MARKS, Shula, « History, the Nation and Empire: Sniping from the Periphery », History Workshop Journal, vol. 29, $\mathrm{n}^{\circ} 1,1990$.

5. Voir par exemple LEVINE, Philippa, « Gendering Decolonisation », Histoire@Politique, vol. 2, n¹1, 2010.

6. Le cas du Cameroun est différent, la république actuelle étant née de l'unification du Cameroun sous tutelle française et d'un des deux territoires sous tutelle britannique, le Cameroun méridional (Southern Cameroons).

7. AShton, Stephen R., «British Government Perspectives on the Commonwealth, 1964-71: An Asset or a Liability? », The Journal of Imperial and Commonwealth History, vol. 35, n¹, 2007. 
8. " conceptually and historically within an international context", " as an ideology and as a form of political practice», MANelA, Erez, The Wilsonian Moment. Self-Determination and the International Origins of Anticolonial Nationalism, Oxford, Oxford University Press, 2007, p. 8.

9. «the norms, interests and objectives of the hegemonic powers - the promotion of peace, prosperity, and "standards of civilization", including democracy, human rights, and environmental protection - that had prevailed», HALL, Ian, "The Revolt against the West: Decolonisation and its Repercussions in British International Thought, 1945-75 ", International History Review, vol. 33, n¹, 2011, p. 55.

10. «Britannic », difficilement traduisible autrement que par «britannique » en français, est employé par opposition à «British» pour proposer une histoire des mondes britanniques qui dépasse les limites du cadre géographique des Iles britanniques et pose différemment la question de la nation, à la fois plus petite (pensons aux quatre «nations » constitutives de l'Etat actuel) et plus large (notamment ici, le monde des dominions, anciennes colonies de peuplement); MCINTYRE, David W., The Britannic Vision. Historians and the Making of the British Commonwealth of Nations, 1907-1948, Basingstoke, Palgrave Macmillan, 2009.

11. http://www.open.ac.uk/researchprojects/makingbritain [consulté le 10.09.2018].

12. " how do particular developments in the global political economy of a specific era shape and/ or become reflected in the dominant social theory ", SCHILLER, Nina Glick, SALAZAR, Noel B., «Regimes of Mobility Across the Globe », Journal of Ethnic and Migration Studies, vol. 39, n², 2013, p. 184.

13. "the seeds of imperialism are imperial, rather than post-colonial ", GRANT, Kevin, LEVINE, Philippa et TRENTMANN, Frank, Beyond Sovereignty: Britain, Empire and Transnationalism, c. 1880-1950, Londres, Palgrave Macmillan, 2007.

14. GORMAN, Daniel, Imperial Citizenship. Empire and the Question of Belonging. Manchester, Manchester University Press, 2006 ; COOPER, Frederick, Citizenship Between Empire and Nation: Remaking France and French Africa, 1945-1960, Princeton, Princeton University Press, 2014.

15. Voir par exemple Rocchi, Jean-Paul et SYLVANISE, Frédéric, «Black Europe: Subject, Struggles and Shifting Perceptions ", Palimpsest, vol. 4, n², 2015.

16. " unfinished, untidy, a mass of aspirations, aspirations and anomalies ", DARWIN, John, The Empire Project, The Rise and Fall of the British World-System, 1830-1970, Cambridge, Cambridge University Press, 2009, p. xi.

17. COOPER, Frederick et STOLER, Ann-Laura, «Between Metropole and Colony: Rethinking a Research Agenda » in Frederick COOPER et Ann-Laura STOLER (ed), Tensions of Empire: Colonial Cultures in a Bourgeois World, Berkeley, University of California Press, 1997, p. 33-34.

18. Voir par exemple SINCLAIR, Georgina, At the End of the Line. Colonial Policing and the Imperial Endgame, 1945-1980, Manchester, Manchester University Press, 2006 ; BELL, Emma, « Normalising the Exceptional: British Colonial Policing Cultures Come Home », Mémoire(s), identité(s), marginalité (s) dans le monde occidental contemporain [En ligne], 10, document 9, 2013, http:// mimmoc.revues.org/1286.

19. La richesse des travaux d'Elizabeth Buettner vient à la fois de l'éventail des pays européens et des territoires coloniaux (ou des anciennes colonies) auxquels elle s'intéresse, et du fait que, si les grands centres impériaux occupant une place importante - la Malaisie, l'Inde, la Rhodésie du Sud ou le Kenya - les aires de l'empire informel sont également prises en considération, de Shanghai à Buenos Aires.

20. Pendant longtemps pour la fin de l'empire britannique en Afrique, la Rhodésie est restée perçue comme un cas exceptionnel qui ne remettait pas réellement en cause une décolonisation « réussie».

21. Usha Iyer, "Decolonisation and the Imperial Cricket conference, 1947-1965: a study in transnational Commonwealth history », Université du Central Lancashire, 2013. 
22. Stuart Whigham, "Scotland's future and 2014: political narratives of the Glasgow Commonwealth Games and the independence referendum », Universite de Loughborough, 2017 ; Jonathan Duckett, "Geographies of youth citizenship and national identity : a case study of Glasgow 2014 Commonwealth Games and the Scottish Independence referendum », Université de Loughborough, 2017.

23. "Volunteering for the Commonwealth Games: what can realist synthesis contribute to health policy making?», Université de Glasgow, 2016. Sur la santé, voir aussi la thèse en médecine de Gerry McCartney, «How will the 2014 Commonwealth Games impact on Glasgow's health, and how will we know? ", Université de Glasgow, 2010.

24. Maureen Kidd, "'Expanding horizons : investigating the Glasgow 2014 legacy for young people in the East End of Glasgow », Université de Glasgow, 2016. Sur ces questions, voir aussi Briony Sharp, "The role of major events in the creation of social legacy: a case study of the Glasgow 2014 Commonwealth Games », Université Queen Margaret, Edinbourg, 2017.

25. Thomas Wilson, «Imagining empire: the design and display strategies of the Imperial Institute and the Commonwealth Institute, 1887-1997 », Université de Brighton, 2016.

26. MBEMBE, Achille, « Qu'est-ce que la pensée postcoloniale ?», Esprit, vol. 12, 2006, p. 125.

27. SIBEUD, Emmanuelle, "Post-colonial et Colonial Studies: enjeux et débats", Revue d'histoire moderne et contemporaine, vol. $51, \mathrm{n}^{\circ} 4 \mathrm{bis}, 2004, \mathrm{p} .88$.

28. "the careful reconstruction of political and economic contexts", DARWIN, John, The Empire Project, op. cit., p. 7.

29. «material, social and cultural resources », COOPER, Frederick, Colonialism in Question. Theory, Knowledge, History, Berkeley, University of California Press, 2005, p. 17.

30. En France, la méfiance, voire l'hostilité, de plusieurs universitaires au courant des études postcoloniales vient en partie de la convergence de deux facteurs malheureux : l'arrivée tardive de ces travaux en France, sous leur forme traduite, et l'absence dans les bibliographies postcoloniales des travaux français en histoire des mentalités, des représentations ou encore de la «politique par le bas", en dehors de quelques références, souvent centrées sur Michel Foucault.

31. SIBEUD, Emmanuelle, «Du postcolonialisme au questionnement postcolonial: pour un transfert critique ", Revue d'histoire moderne et contemporaine, vol. 54, $\mathrm{n}^{\circ} 4,2007$, p. 148.

32. " a Eurocentric critique of Eurocentrism », «studies about the subaltern rather than studies with and from a subaltern perspective», GROSFOGUEL, Ramón, «Decolonizing Post-Colonial Studies and Paradigms of Political-Economy: Transmodernity, Decolonial Thinking and Global Coloniality ", Transmodernity: Journal of Peripheral Cultural Production of the Luso-Hispanic World, vol. $1, \mathrm{n}^{\circ} 1,2011$.

33. LEWIS, Martin W., WIGEN, Kären, The Myth of Continents. A Critique of Metageography, Berkeley, University of California Press, 1997.

34. Voir CHAKRABARTY, Dipesh, Provincializing Europe: Postcolonial Thought and Historical Difference, Princeton, Princeton University Press, 2000 ; CHATTERJEE, Partha, The Black Hole of Empire: History of a Global Practice of Power, Princeton, Princeton University Press, 2012.

35. Аүоов, Mohammed, "Inequality and theorizing in international relations: the case for subaltern realism ", International Studies Review, vol. 4, n³, 2002, p. 27-48. Pour les limites du concept, voir BARNETT, Michael, «Radical chic? Subaltern realism : a rejoinder », International Studies Review, vol. 4, n³, p. 49-62.

36. SHARP, Joanne, «Subaltern geopolitics : introduction », Geoforum, vol. 42, n³, 2011.

37. CRAGGS, Ruth, "Subaltern geopolitics and the post-colonial Commonwealth, 1965-1990", Political Geography, ${ }^{\circ} 65,2018$.

38. MCCONNELL, Fiona, "Liminal geopolitics : the subjectivity and spatiality of diplomacy at the margins », Transactions of the Institute of British Geographers, vol. 41, n²1, 2017. 
39. SHIMAZU, Naoko, «Diplomacy as theatre : staging the Bandung Conference of 1955 ", Modern Asian Studies, vol. 48, n 2014 ; voir aussi SHIMAZU, Naoko, «Places in Diplomacy ", Political Geography, vol. 31, n6, 2012.

40. oWEN, Nicholas, «Four Straws in the Wind : Metropolitan Anti-Imperialism, January-February 1960 » in L.J. BUTLER et Sarah STOcкWELl (ed), The Wind of Change: Harold Macmillan and British Decolonization, Oxford, Oxford University Press, 2013.

41. CRAGGS, Ruth, "Towards a political geography of hotels: Southern Rhodesia, 1958-1962 ", Political Geography, vol. 31, $\mathrm{n}^{\circ} 4,2012$.

42. BROWNELL, Josuah, " "A sordid tussle on the Strand": Rhodesia House during the UDI rebellion (1965-80) », Journal of Imperial and Commonwealth History, vol. 38, 2010.

43. MCGREGOR, JoAnn, "Locating exile: decolonization, anti-imperial spaces and Zimbabwean students in Britain, 1965-1980 », Journal of Historical Geography, vol. 57, 2017.

44. Ibid., p. 65.

45. «the globalising processes of the post-1945 period were rooted in the armed conflicts of decolonisation ", "defined new normative standards of anti-state violence by non-state actors and groups ", тномаs, Martin et тномеsоn, Andrew, "Empire and Globalisation: From "High Imperialism" to Decolonisation ", International History Review, vol. 36, n²1, 2014, p. 144.

46. L'étude menée par la Royal Commonwealth Society en 2010 sur la perception du Commonwealth à travers l'ensemble des pays membres n'a pas pour l'instant été actualisée - et était par ailleurs le fruit du travail d'une organisation directement liée au Commonwealth.

47. "suggests both contingency and movement", GILROY, Paul, After Empire. Melancholia or Convivial Culture? Londres, Routledge, 2004, p. xii.

48. GROSSER, Pierre, "L'histoire mondiale/globale, une jeunesse exubérante mais difficile», Vingtième Siècle, vol. 110, $\mathrm{n}^{\circ} 2,2011$, p. 8.

49. FERGUSON, Niall, Empire: How Britain Made the Modern World, Londres, Allen Lane, 2003.

50. RÉVAUGER, Jean-Paul, "La Grande-Bretagne de Tony Blair entre deux modèles de société ", Observatoire de la société britannique, vol. 1, 2006.

51. Cela concerne aussi bien le Togo, qui est candidat au statut de membre, que le Japon, dont plusieurs hommes politiques (britanniques notamment) estiment de temps à autre que son admission serait souhaitable.

52. REDONNET, Jean-Claude, Le Commonwealth. Politiques, coopération et développement anglophones, Paris, Presses universitaires de France, 1998, p. 235.

53. Voir BARDOLPH, Jacqueline et al., Oppression et expression dans la littérature et le cinéma : Afrique, Amérique, Asie, Paris, L'Harmattan, 1981.

54. MBEMBE, Achille, De la postcolonie, Paris, Karthala, 2000, p. 20.

55. HARROW, Kenneth et MPOCHE, Kitizus (dir.), Language, Literature and Education in Multicultural Societies: Collaborative Research on Africa. Newcastle, Cambridge Scholars Publishing, 2008.

56. АКО, Edward O., "From Commonwealth to Postcolonial Literature », CLCWeb: Comparative Literature and Culture, vol. 6, n², 2004.

57. NEWELL, Stephanie, "Afterword » in Derek R. PETERSON, Emma HUNTER et Stephanie NEWELL (dir.), African Print Cultures. Newspapers and their Publics in the Twentieth Century, Ann Arbor, University of Michigan Press, 2016.

58. NEWELL, Stephanie, Literary Culture in Colonial Ghana. "How to play the game of life", Manchester, Manchester University Press, 2002.

59. Voir par exemple VAN DAMME, Stéphane, "Comprendre les Cultural Studies: une approche d'histoire des savoirs ", Revue d'histoire moderne et contemporaine, vol. 51, n4bis, 2004.

60. «beyond certain triumphalist discourses of a globalized, Anglophone uniformity in order to understand better the complexity and diversity - linguistic, cultural, political - of the world in which we live ", FORSDICK, Charles et MURPHY, David, «Introduction: the Case for Francophone 
Postcolonial Studies » in Charles FORSDICK et David MURPHY (ed), Francophone Postcolonial Studies. A Critical Introduction, Londres, Arnold, 2003, p. 14.

61. onslow, Sue (dir.), "The Commonwealth and Media Freedom», The Round Table: The Commonwealth Journal of International Affairs, vol. 107, $\mathrm{n}^{\circ} 2,2018$.

62. An., « Editorial », The Journal of Commonwealth Political Studies, vol.1, n¹, 1961.

63. DE SMITH, S.A., «Westminster's export models: the legal framework of responsible government », The Journal of Commonwealth Political Studies, vol. 1, n¹, 1961.

64. Kumarasingham, Harshan, A Political Legacy of the British Empire: Power and the Parliamentary System in Post-Colonial India and Sri Lanka, Londres, I.B. Tauris, 2013.

65. FRANK, Robert, "Une histoire problématique: une histoire du temps présent ", Vingtième Siècle. Revue d'histoire, vol. 71, 2001, p. 86.

66. Voir http://www.commonwealthoralhistories.org. Cette base complète d'autres archives d'entretiens précieuses, comme celles de l'Anti-Apartheid Movement, ou le British Diplomatic Oral History Programme.

67. Derrick McKoy, «The emerging regimes on anticorruption and state enterprise governance in the Commonwealth Caribbean ", Université de Leicester, 2010; Kyela Leakey, "The role of the Chief Justice in Commonwealth Africa: a comparative study of South Africa, Ghana and Kenya ", Queen Mary, Université de London, 2012; Izevbuwa Ayela-Ikhimiukor, « Assessing the impact of contemporary international criminal courts and tribunals in the Commonwealth », Universite du Middlese, 2015; Jason Haynes, «Re-thinking anti-trafficking law and practice: European and Commonwealth Caribbean perspectives », Université de Durham, 2015; Mahalia Jackman, « Living in Sodom's shadow : essays on attitudes towards gay men and lesbians in the Commonwealth Caribbean ", Université de Manchester, 2017.

68. Dans les dix thèses soutenues en histoire au Royaume-Uni entre 2008 et 2018 et portant spécifiquement sur le Commonwealth, l'internationalisme, le transnationalisme ou les transferts occupent une place centrale.

69. Voir par exemple LYDON, Ghislaine, On Trans-Saharan Trails. Islamic Law, Trade Networks and Cross-Cultural Exchange in Nineteenth-Century Western Africa, Cambridge, Cambridge University Press, 2009; MCDougall, James, scheele, Judith, Saharan Frontiers. Space and Mobility in Northwest Africa, Bloomington, Indiana University Press, 2012.

70. Select Committee on Soft Power and the UK's Influence, « First Report: Persuasion and Power in the Modern World", 11 mars 2014, http://www.publications.parliament.uk/pa/ld201314/ ldselect/ldsoftpower/150/15002.htm (consulté le 3 juillet 2016).

71. MURPHY, Philip, The Empire's New Clothes: The Myth of the Commonwealth, Londres, Hurst, 2018.

72. " no two leaders now - probably - have that ability to self-reference across a common past of some kind ", Sandra Pepera, Entretien avec Sandra Pepera, 7 août 2014, Commonwealth Oral History Project (www.commonwealthoralhistories.org), https://sas-space.sas.ac.uk/5817/1/ Sandra_Pepera_Transcript.pdf [consulté le 10.09.2018] p. 27.

\section{RÉSUMÉS}

Cet article propose non pas de retracer l'histoire du Commonwealth contemporain mais plutôt de montrer comment le dialogue entre disciplines a permis un renouvellement de l'écriture de cette histoire, et dans quelle mesure le Commonwealth des Nations, comme espace géopolitique, 
acteur international multilatéral et objet d'imaginaires politiques divers, constitue un prisme d'analyse critique pertinent pour l'histoire - internationale, transnationale et connectée - du monde contemporain. Il conclut que pour la recherche, le dialogue interdisciplinaire permet de maintenir l'équilibre entre le Commonwealth comme idée, comme pratique et comme institution (trois aspects qui évoluent dans le temps et que différents acteurs formulent, mettent en œuvre et interprètent différemment), afin de proposer une étude des relations internationales dans toute leur épaisseur.

This article does not propose to retrace the history of the contemporary Commonwealth but rather to show how the dialogue across disciplines has renewed the history of the organisation and the extent to which the Commonwealth of Nations, as a geopolitical space, a multilateral international actor and the object of diverse political imaginaries, is a relevant critical tool for the (international, transnational and connected) history of the contemporary world. It concludes that for research, interdisciplinary dialogue is a means of achieving some balance between the Commonwealth as an idea, as practice and as institution (three aspects which evolve over time and that different actors formulate, implement and interpret in various ways), in order to propose a study of international relations in all their depth.

\section{INDEX}

Mots-clés : Commonwealth, diplomatie, globalisation, normes, postcolonial

Keywords : Commonwealth, diplomacy, globalisation, norms, postcolonial

\section{AUTEUR}

\section{MÉLANIE TORRENT}

Université Paris Diderot, Laboratoire de recherches sur les cultures anglophones (LARCA, UMR 8225) 